\title{
Hepatocellular carcinoma with inferior vena caval and right atrial tumor thrombi and massive pulmonary artery embolism: A case report
}

\author{
JIAN HUANG $^{1 *}$, ZE-YA PAN $^{1 *}$, LI LI $^{2^{*}}$, BEI-GE JIANG ${ }^{1}$, FANG-MING GU $^{1}$, \\ ZHEN-GUANG WANG $^{1}$, ZHI-HONG WANG ${ }^{1}$ and WEI-PING ZHOU ${ }^{1}$
}

\begin{abstract}
${ }^{1}$ Third Department of Hepatic Surgery, Eastern Hepatobiliary Surgery Hospital, Second Military Medical University, Shanghai 200438; ${ }^{2}$ Department of Cardiology, The First Affiliated Hospital of Nanchang University, Nanchang University, Nanchang, Jiangxi 330000, P.R. China
\end{abstract}

Received February 12, 2016; Accepted October 10, 2016

DOI: $10.3892 / \mathrm{mco} .2016 .1093$

\begin{abstract}
The aim of the present study was to report the case of a 55 -year-old female patient with a sizeable $(7.1 \times 6.2 \mathrm{~cm})$ hepatocellular carcinoma (HCC), who succumbed to massive pulmonary artery embolism. The main symptoms included sudden thoracodynia, dyspnea and transient coma. The initial diagnosis was HCC according to the typical abdominal ultrasound and triple-phase abdominal computed tomography (CT) findings, chronic hepatitis B infection and elevated $\alpha$-fetoprotein levels (1,036 $\mu \mathrm{g} / \mathrm{l}$; normal, $0-20 \mu \mathrm{g} / \mathrm{l})$. Two days following admission, the patient developed recurrent chest pain and shortness of breath. The electrocardiogram and myocardial enzyme levels were normal, but the D-dimer level was elevated to 7,210 $\mu \mathrm{g} / 1$ (normal, 0-550 $\mu \mathrm{g} / \mathrm{l}$ ). Magnetic resonance angiography and a contrast-enhanced chest CT confirmed that the inferior vena cava and right atrium were invaded by tumor thrombi; the bilateral pulmonary embolism was also suspected to be formed by tumor thrombi. The final diagnosis was HCC with inferior vena caval and right atrial tumor thrombi, as well as massive pulmonary embolism. Anticoagulation therapy with low-molecular weight heparin calcium was administered; however, the patient succumbed to pulmonary embolism in $<2$ months.
\end{abstract}

Correspondence to: Professor Ze-Ya Pan, Third Department of Hepatic Surgery, Eastern Hepatobiliary Surgery Hospital, Second Military Medical University, 225 Chang Hai Road, Yang Pu, Shanghai 200438, P.R. China

E-mail: panzyhj@163.com

${ }^{*}$ Contributed equally

Key words: hepatocellular carcinoma, pulmonary arterial embolism, anticoagulant therapy, contrast-enhanced chest computed tomography, D-dimer

\section{Introduction}

Invasion of the hepatic vein, inferior vena cava (IVC) and pulmonary artery is a conspicuous characteristic in the majority of advanced cases of hepatocellular carcinoma (HCC). Once pulmonary artery thrombosis occurs, most patients succumb to respiratory failure. The major type of malignant tumor in developing regions is HCC, particularly in China (1). The prognosis is very poor in the advanced stages of the disease, possibly due to tumor vascular invasion. HCC patients with pulmonary embolism and IVC invasion have been reported to survive for $\leq 2$ months without surgery (2). We herein present the case of a patient with HCC with a sizeable primary tumor, invasion of the IVC and right atrium by tumor thrombi and massive bilateral pulmonary embolism.

\section{Case report}

On April 18, 2015, a 55-year-old female patient presented to the Eastern Hepatobiliary Surgery Hospital with a 6-h history of sudden thoracodynia, dyspnea and transient coma. The oxygen partial pressure was $49.9 \mathrm{mmHg}$, the carbon dioxide partial pressure was $30.9 \mathrm{mmHg}$ and the blood oxygen saturation was $86.4 \%$ ( $\mathrm{pH} 7.52$ ). Routine abdominal ultrasound revealed a large low-density mass in the right hepatic lobe. The patient had chronic hepatitis B for 40 years; there was no history of diabetes, coronary heart disease or hypertension.

Physical examination on admission revealed a body temperature of $36.8^{\circ} \mathrm{C}$, a heart rate of 100 beats $/ \mathrm{min}$, a blood pressure of 103/67 mmHg, a respiratory rate of 20 breaths/min and an Eastern Cooperative Oncology Group performance status of 2. The patient had no ascites, edema, signs of hepatic failure or stigmata of cirrhosis. The results of the laboratory tests were as follows: Hemoglobin, $143 \mathrm{~g} / 1$ (normal, 115-150 g/l); leukocyte count, $4.55 \times 10^{9} / 1$ (normal, 3.5-9.5 $\left.\times 10^{9} / 1\right)$; platelet count, $243 \times 10^{9} / 1$

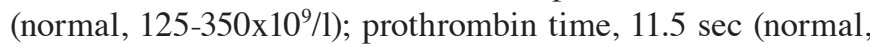
$11-13 \mathrm{sec}$ ); activated partial thromboplastin time, $27.7 \mathrm{sec}$ (normal, 24-37 sec); total bilirubin, $9.3 \mu \mathrm{mol} / 1$ (normal, 3.42-20.52 $\mu \mathrm{mol} / \mathrm{l}$ ); albumin, $39.2 \mathrm{~g} / 1$ (normal, 40-55 g/l); aspartate aminotransferase, $63 \mathrm{U} / 1$ (normal, 13-35 U/1); alanine aminotransferase, $45 \mathrm{U} / 1$ 
(normal, 7-40 U/1); $\gamma$-glutamyl transpeptidase, $113 \mathrm{U} / 1$ (normal, 7-45 U/1); $\alpha$-fetoprotein (AFP), 1,036 $\mu \mathrm{g} / 1$ (normal, 0-20 $\mu \mathrm{g} / \mathrm{l}$ ); abnormal prothrombin, 3,168 mAU/ml (normal, 0-40 mAU/ml); hepatitis B surface antigen positivity; hepatitis B e antibody positivity; hepatitis B core antibody positivity; and hepatitis B virus DNA levels of 7.08x10 $1 \mathrm{IU} / \mathrm{ml}$ (normal, $<50 \mathrm{IU} / \mathrm{ml}$ ). Blood gas analysis revealed an oxygen partial pressure of $112 \mathrm{mmHg}$, a carbon dioxide partial pressure of $36 \mathrm{mmHg}$ and a blood oxygen saturation of $99 \%$ ( $\mathrm{pH} 7.44)$. The electrocardiogram (ECG) displayed a normal sinus rhythm. Chest radiography and echocardiography showed no abnormalities; however, triple-phase abdominal computed tomography (CT) revealed a sizeable lesion $(7.1 \times 6.2 \mathrm{~cm})$ in the right hepatic lobe (Fig. 1A), along with thrombosis of the IVC (Fig. 1B). The lesion exhibited typical arterial phase enhancement and venous/delayed phase washout; based on these findings, the patient was diagnosed with HCC.

Two days after admission, the patient developed recurrent chest pain and shortness of breath, with a blood oxygen saturation of $90 \%$ (normal, 95-99\%). Urgent blood gas analysis revealed an oxygen partial pressure of $78 \mathrm{mmHg}$ (determined under oxygen inhalation via nasal prongs at $3 \mathrm{l} / \mathrm{min}$ ) and the D-dimer was elevated to 7,210 $\mu \mathrm{g} / 1$ (normal, 0-550 $\mu \mathrm{g} / \mathrm{l}$ ). The ECG revealed nodal tachycardia and no abnormal wave shapes. The myocardium zymogram examination and M-mode echocardiography results were normal; thus, acute myocardial infarction was excluded. Urgent magnetic resonance angiography (MRA) revealed that the IVC (Fig. 2A and B) and right atrium (Fig. 2C) were invaded by tumor thrombi.

Routine chest CT examination was normal (Fig. 3A) despite the patient's history of chest pain and shortness of breath. Considering that hematogenous metastasis is a characteristic feature of HCC, pulmonary artery metastasis was suspected and an urgent contrast-enhanced CT scan was performed, revealing nodular filling defects in the main pulmonary arteries (Fig. 3B), and the diagnosis of bilateral pulmonary emboli without lung metastasis was considered.

Based on the aforementioned findings, the final diagnosis was HCC with IVC and right atrial tumor thrombi, as well as massive pulmonary embolism. The patient was administered palliative treatment due to the advanced stage of the disease according to the Barcelona Clinic Liver Cancer Classification (3). To prevent further formation and extension of blood thrombi, anticoagulant therapy with low-molecular weight heparin calcium was administered (4,100 IU q12h). The chest pain and shortness of breath resolved, a routine blood test and coagulation function test revealed no abnormalities, and the D-dimer level exhibited a decreasing trend. The patient was in a stable condition following anticoagulant treatment for 3 days and was discharged from the hospital. Anticoagulant therapy with low-molecular weight heparin calcium was continued at another local hospital, but he patient eventually succumbed to sudden pulmonary thrombosis within 2 months after being discharged, as we were informed by her family.

\section{Discussion}

Primary liver cancer is the fifth most commonly diagnosed type of cancer and the second leading cause of cancer-related mortality in men, whereas it is the ninth most commonly diagnosed type of cancer and the second leading cause of

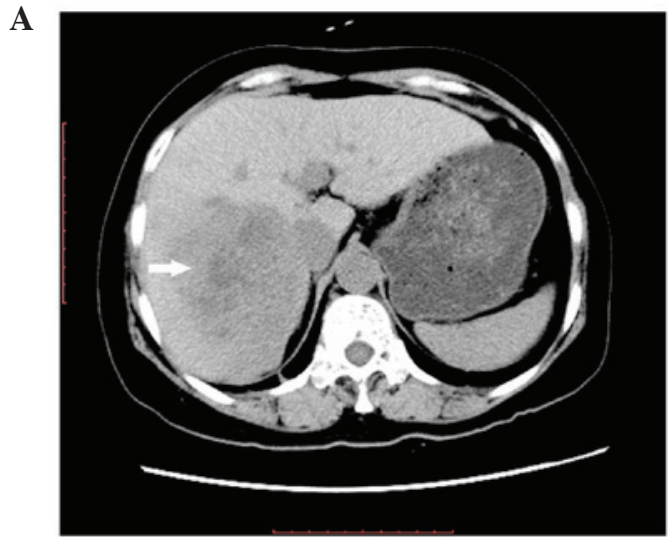

B

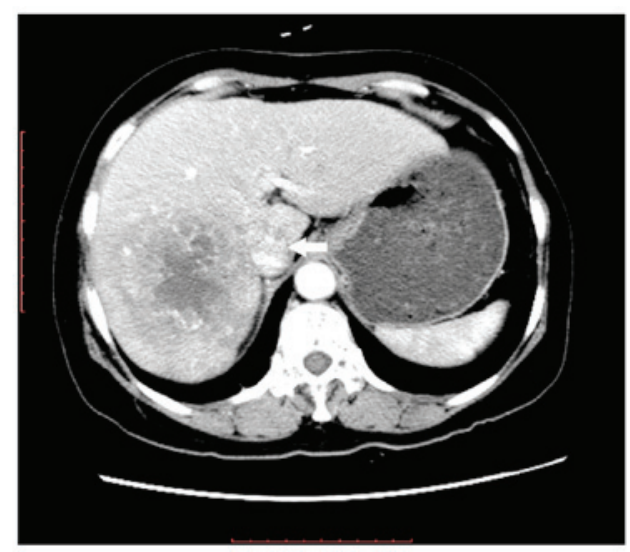

Figure 1. Triple-phase abdominal computed tomography showing (A) a large low-density lesion, sized $7.1 \times 6.2 \mathrm{~cm}$, in the right liver lobe (arrow) and (B) a massive tumor thrombus in the inferior vena cava (arrow).

cancer-related mortality in women worldwide; HCC accounts for the majority of primary liver cancers (4). The diagnosis of $\mathrm{HCC}$ is typically determined by radiological liver imaging in combination with serum AFP without the need for biopsy, as there is a consensus that bioptic proof of HCC is not required in patients with chronic hepatitis B infection or cirrhosis and non-invasive criteria may be applied $(5,6)$. Therefore, in our patient, the diagnosis of HCC was established beased on the 40-year history of chronic hepatitis B, the high levels of serum AFP and the radiological findings, which were typical of HCC.

Advanced HCC generally metastasizes to the lungs, bone, brain and adrenal glands. Vascular invasion and tumor thrombosis formation are prominent characteristics in the majority of cases of advanced HCC due to activation of hemostasis $(7,8)$. The incidence of vascular invasion increases with increasing tumor size and it has been reported to be $82 \%$ in patients with serum AFP levels $>1,000 \mu \mathrm{g} / 1$ and a tumor diameter of $>5 \mathrm{~cm}$ (9). The most frequent type of tumor thrombosis in HCC patients is portal vein thrombosis (20-65\%), followed by systemic venous thromboembolism $(6 \%)(8,10)$. The incidence of hepatic vein thrombosis in HCC patients is 1.4-4.9\% (11). Tumor thrombosis from HCC invading the hepatic vein occasionally spreads to the IVC and even the right atrium, which has been reported in $0.67-3 \%$ of the cases $(7,12)$. In the present case, although the ECG revealed no abnormalities, MRA revealed a mass extending from the IVC into the right atrium. Based on the findings of a previous case study, which demonstrated specific uptake of the contrast 


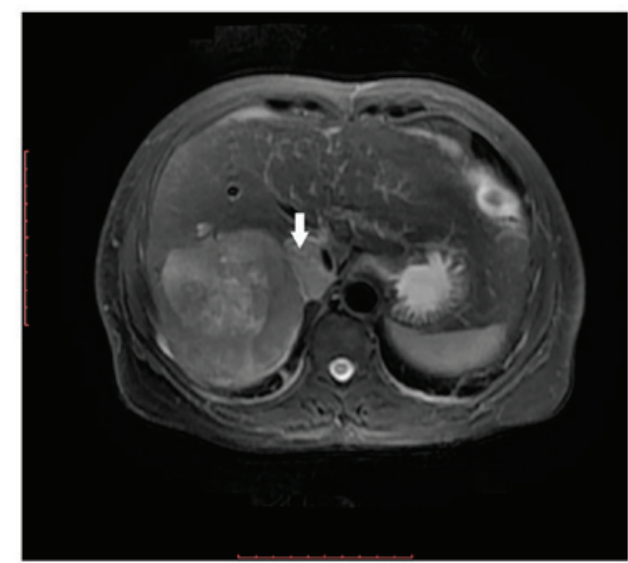

B

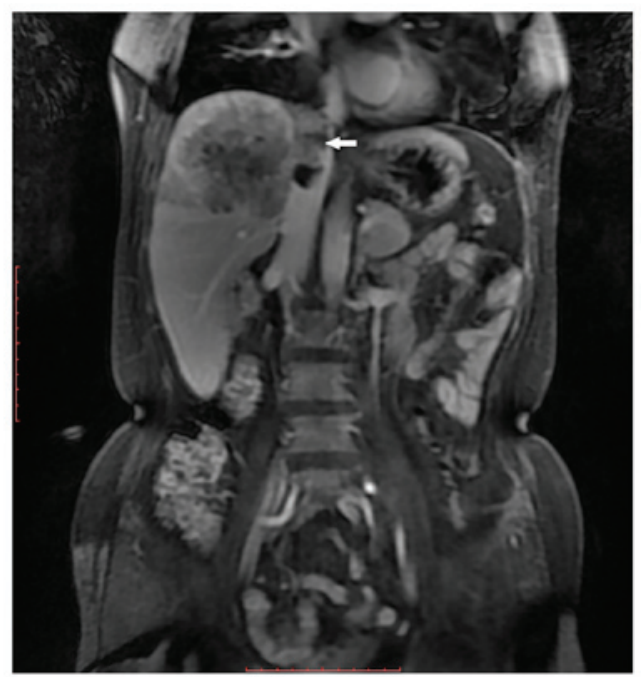

C

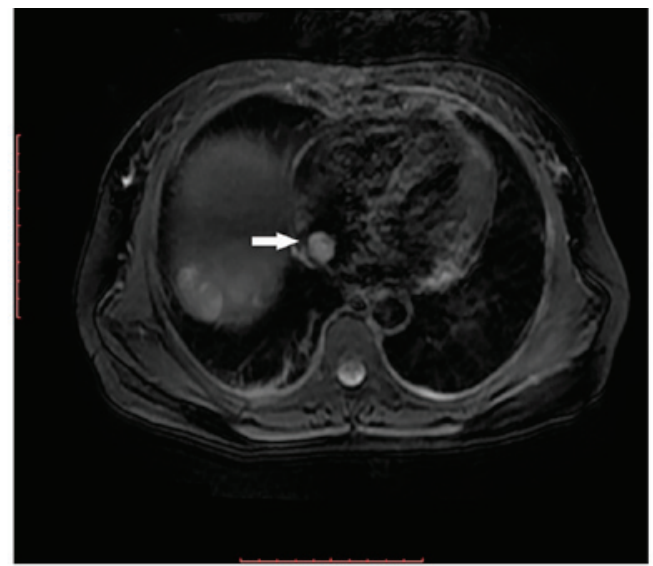

Figure 2. MRA scans. (A and B) MRA scans showing an enlarged liver, with an extensive heterogenous lesion with necrosis in the right lobe, thrombosis in the inferior vena cava (shown by the white arrows) and (C) invasion of the right atrium by tumor thrombus (shown by the arrow). MRA, magnetic resonance angiography.

medium gadolinium by tumor tissue and not the thrombus, helped confirm that the masses in the IVC and right atrium were indeed tumor thrombi (13). Intra-atrial growth of HCC may not cause any symptoms per se, but may lead to pulmonary thrombosis and pulmonary metastasis $(14,15)$. Primary thrombi in the right atrium are usually immobile and attached to the atrial wall; however, secondary thrombi are mobile and may temporarily lodge into the right atrium; thus, thrombi in the right atrium may
A

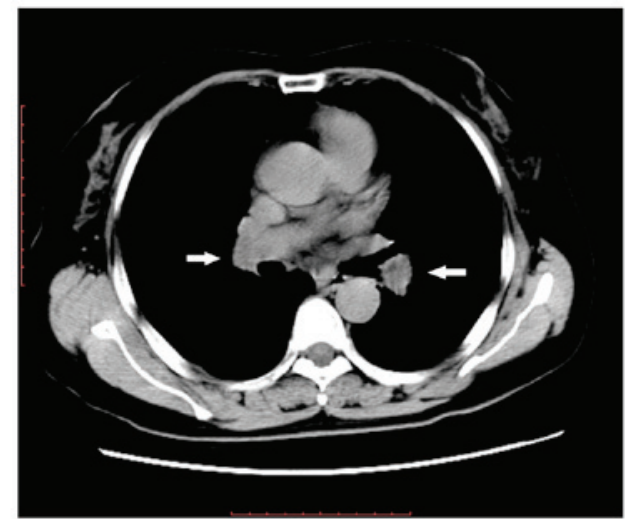

B

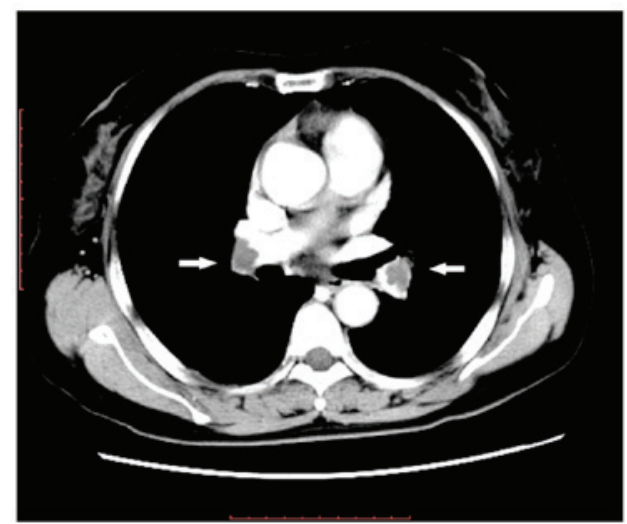

Figure 3. Computed tomography (CT) scans. (A) A routine CT without contrast enhancement hardly showed obvious abnormalities in the two pulmonary arteries (arrows). (B) Massive bilateral pulmonary artery thrombosis (arrows) on chest contrast-enhanced CT (the maximum diameter of the pulmonary artery and the thrombus was 19.3 and $13.8 \mathrm{~mm}$, respectively).

enter the pulmonary artery via the blood flow (10). In the present case, it was hypothesized that the masses in the pulmonary artery were tumor thrombi originating from the right atrium, due to the diagnosis of HCC, the sudden occurrence of the symptoms and the absence of edema of the lower limbs, excluding the possibility of deep venous thrombosis.

In the majority of the patients, the symptoms of pulmonary embolism include dyspnea, chest pain, pre-syncope or syncope, cough, fever and/or hemoptysis (16). Although these symptoms are non-specific, once chest pain and shortness of breath appear in HCC patients in the absence of underlying cardiopulmonary diseases, pulmonary embolism should be highly suspected.

In the present case, the sudden onset of thoracodynia, dyspnea and transient coma, a significant reduction of partial oxygen pressure and a marked rise of D-dimer levels should draw clinicians' attention to the possibility of HCC with pulmonary tumor thrombosis. Although a chest X-ray revealed no abnormalities, a contrast-enhanced chest CT clearly demonstrated massive bilateral pulmonary artery thrombosis; however, a previous routine CT without contrast enhancement could not distinguish between tumor thrombosis and the surrounding tissues due to their similar density. This illustrates the importance of the chest $\mathrm{CT}$ examination with contrast enhancement, without which the diagnosis of pulmonary embolism would have been missed, with a high risk of tumor thrombi being dislodged during subsequent hepatectomy and 
tumor thrombosis resection. Indeed, the patient succumbed to pulmonary embolism within 2 months.

HCC patients with IVC invasion and right atrial tumor thrombi usually have poor survival, owing to the difficulty of early diagnosis (17). Resection may be the only treatment available for tumor thrombi in patients with HCC (17). According to a previous study, the 1-year survival rate among HCC patients with pulmonary embolism and IVC invasion who received surgery was $40 \%$; however, in the group without surgery, the median survival time was only 3 days and none of the patients survived for $>2$ months (2). In the present case, the patient and her family refused surgery due to the high associated risk, although they were informed that surgery may prolong the patient's survival.

In conclusion, three points are important in the present case: First, in patients with HCC and tumor thrombus of the hepatic vein or IVC, symptoms of respiratory distress and chest pain should raise the suspicion of pulmonary embolism. Second, a significant increase in D-dimer levels has a laboratory diagnostic value (18); in addition, routine thoracic contrast-enhanced $\mathrm{CT}$ is necessary, as routine chest CT and X-ray cannot illustrate extensive pulmonary embolism, resulting in misdiagnosis with severe consequences. Finally, despite three events of pulmonary embolism affecting large areas, the patient in the present study still survived for $\sim 2$ months. This may be attributed to the anticoagulation effect of the low-molecular weight heparin calcium, which may prevent immediate further formation and extension of blood thrombi, as well as promote the collateral circulation around pulmonary arteries via peripheral blood vessels. Palliative treatment may prolong patient survival, although the prognosis for HCC patients with vascular invasion is generally poor.

\section{Acknowledgements}

The authors are grateful for the support of the Innovation Program of Shanghai Municipal Education Commission (grant no. 12ZZ077).

\section{References}

1. Ferlay J, Soerjomataram I, Dikshit R, Eser S, Mathers C, Rebelo M, Parkin DM, Forman D and Bray F: Cancer incidence and mortality worldwide: Sources, methods and major patterns in GLOBOCAN 2012. Int J Cancer 136: E359-E386, 2015.

2. Lin HH, Hsieh CB, Chu HC, Chang WK, Chao YC and Hsieh TY: Acute pulmonary embolism as the first manifestation of hepatocellular carcinoma complicated with tumor thrombi in the inferior vena cava: Surgery or not. Dig Dis Sci 52: 1554-1557, 2007.
3. Llovet JM, Brú C and Bruix J: Prognosis of hepatocellular carcinoma: The BCLC staging classification. Semin Liver Dis 19: 329-338, 1999.

4. Torre LA, Bray F, Siegel RL, Ferlay J, Lortet-Tieulent J and Jemal A: Global cancer statistics, 2012. CA Cancer J Clin 65: 87-108, 2015.

5. Lau WY and Lai EC: Hepatocellular carcinoma: Current management and recent advances. Hepatobiliary Pancreat Dis Int 7: 237-257, 2008.

6. Bruix J and Sherman M; American Association for the Study of Liver Diseases: Management of hepatocellular carcinoma: An update. Hepatology 53: 1020-1022, 2011.

7. Masci G, Magagnoli M, Grimaldi A, Covini G, Carnaghi C, Rimassa L and Santoro A: Metastasis of hepatocellular carcinoma to the heart: A case report and review of the literature. Tumori 90: 345-347, 2004.

8. Connolly GC, Chen R, Hyrien O, Mantry P, Bozorgzadeh A, Abt $\mathrm{P}$ and Khorana $\mathrm{AA}$ : Incidence, risk factors and consequences of portal vein and systemic thromboses in hepatocellular carcinoma. Thromb Res 122: 299-306, 2008.

9. Sakata J, Shirai Y, Wakai T, Kaneko K, Nagahashi M and Hatakeyama K: Preoperative predictors of vascular invasion in hepatocellular carcinoma. Eur J Surg Oncol 34: 900-905, 2008.

10. Panduranga P, Al-Mukhaini M, Ratnam L and Al-Harthy S: Mobile right atrial thrombus with pulmonary thromboembolism in a patient with advanced hepatocellular carcinoma and disseminated tumor thrombosis. Heart Views 12: 173-177, 2011.

11. Zhang YF, Wei W, Guo ZX, Wang JH, Shi M and Guo RP: Hepatic resection versus transcatheter arterial chemoembolization for the treatment of hepatocellular carcinoma with hepatic vein tumor thrombus. Jpn J Clin Oncol 45: 837-843, 2015.

12. Afonso DV, Laranjeira A, Galrinho A and Fragata J: Metastatic hepatocellular carcinoma: Right atrial tumor as primary clinical manifestation. Case report. Rev Port Cir Cardiotorac Vasc 15: 79-81, 2008 (In Portuguese).

13. Lazaros G, Samara C, Nikolakopoulou Z and Tassopoulos N: Growth of hepatocellular carcinoma into the right atrium. A case of antemortem diagnosis with magnetic resonance imaging of the heart. Acta Cardiol 58: 563-565, 2003.

14. Pellicelli AM, Barba J, Gomez AJ and Borgia MC: Echocardiographic follow-up of right atrial tumoral invasion by hepatocarcinoma: A case report. Cardiologia 37: 151-153, 1992.

15. Martínez Baca-López F, Ramírez-Arias E, Rayas-Gómez AL, Bernal-Ruiz EA and Saturno-Chiu G: Hepatocellular carcinoma with invasion into right cardiac cavities: Report of a case and literature review. J Am Soc Echocardiogr 17: 192-194, 2004.

16. Konstantinides SV, Torbicki A, Agnelli G, Danchin N, Fitzmaurice D, Galiè N, Gibbs JS, Huisman MV, Humbert M, Kucher N, et al: 2014 ESC guidelines on the diagnosis and management of acute pulmonary embolism. Eur Heart J 35: 3033-3069, 3069a-3069k, 2014.

17. Fujisaki M,Kurihara E, Kikuchi K, Nishikawa K and Uematsu Y: Hepatocellular carcinoma with tumor thrombus extending into the right atrium: Report of a successful resection with the use of cardiopulmonary bypass. Surgery 109: 214-219, 1991.

18. Torbicki A, Perrier A, Konstantinides S, Agnelli G, Galiè N, Pruszczyk P, Bengel F, Brady AJ, Ferreira D, Janssens U, et al: Guidelines on the diagnosis and management of acute pulmonary embolism: The task force for the diagnosis and management of acute pulmonary embolism of the European Society of Cardiology (ESC). Eur Heart J 29: 2276-2315, 2008. 